\title{
Assessment of Dangerous Traffic Situations for Autonomous Vehicles
}

\author{
Péter Galántai1* \\ ${ }^{1}$ Department of Control for Transportation and Vehicle Systems, Faculty of Transportation and Engineering and Vehicle \\ Engineering, Budapest University of Technology and Economics, H-1521 Budapest, P.O.B. 91, Hungary \\ * Corresponding author, e-mail: peti288@icloud.com
}

Received: 08 February 2020, Accepted: 11 February 2020, Published online: 08 March 2022

\begin{abstract}
This article will show some dangerous situations with autonomous vehicles. The main question in this article is how does the ACC (Adaptive Cruise Control) as an autonomous vehicle function work? In which cases must the driver act and how. The research showed some real life examples based on a Euro NCAP test. The car manufacturers say that the modern ACC systems can be well used in urban traffic, especially in traffic jam, but there can be some critical situations which can cause an accident if the driver does not act. These situations are collected in this article. The main aim of the article is to identify a methodological framework for estimating the proper operation conditions related to the analyzed automated driving assistant systems.
\end{abstract}

\section{Keywords}

ACC, automated driving assistant systems, autonomous vehicles, urban traffic, dangerous situations

\section{Introduction}

Today's traffic can be characterized by intensive spread of automated systems and vehicle functions. Among other research orientations coordinated by the automotive industry, the development of driving technology is extremely crucial. Related to this topic it is important to emphasize that the testing of vehicles with high level of automation can now be carried out on the public roads of Hungary. In addition, many vehicles are already on the roads that are equipped with automated driving assistant systems. How much can we rely on these systems in everyday traffic? In what circumstances do these systems require the driver to intervene? What are the factors that can result in an intervention and what would happen without the driver's intervention? What can and what cannot be required from an automated driving assistant system? These are the questions, which are tried to be answered by this article (Mandžuka, 2018).

The main aim of the article is to identify a methodological framework for estimating the proper operation conditions related to the analyzed automated driving assistant systems. Based on the application of the developed test assessment method, those cases have been defined, where it is proved to be likely that the driver needs to intervene the operation of the automated function. In addition, those operation conditions have been defined where the performance of the system is not acceptable. Furthermore the main goal of the analysis is to investigate and evaluate errors that have been experienced during the usage of automated driving assistant systems (Galba et al., 2015).

\section{Idea of the research}

The main research orientations of the model development phase have been identified based on the experiences gained from the car rental sector. The cars typically had 4 functions, such as parking assistant, lane guidance, active cruise control, and emergency brake assistant (AEB). Most of the opportunities were to test the active cruise control (paired with automatic transmission), which is an upgraded version that can work in a traffic jam, thus to stop behind the car in front and then, if it starts, to continue to follow. After standing for more than a few seconds, the driver has to restart the car by pressing the RES button or pressing the accelerator pedal (this is because traffic situation may have changed, so it's important for the driver to decide if the car can start). It can be very useful in an afternoon traffic jam, and in my opinion, this driver support feature can also have a beneficial effect on driver behavior. However, the practice also confirmed that the driver should not rely solely on the car for its intervention 
if necessary. Based on the experienced conflict situations, three critical situations requiring intervention have been classified:

1. The car stopped at a red light, a tall, high roof van stopped in front of the car, but there was no reaction from the ACC and the car didn't slow down, so the driver had to brake the car intensively to avoid a collision before the car ran into it.

2. On a double-lane road, the car was driven with the $\mathrm{ACC}$ in the inner lane at the allowed $50 \mathrm{~km} / \mathrm{h}$, while a car passing in front of it suddenly dropped a bicycle in the outer lane. Within a very close stopping distance, the car ahead moved from the other lane (about $1 / 4$ of the width of the test car). However, there was no braking performed by the ACC, so the driver had to brake in order to avoid a roll-over accident that might have occurred from the situation that had developed.

3. A 2-lane road turned left from a red light on a large curve to a 3-lane road. At the red light, the car stopped behind another car, when the light getting green the car started also such the other cars in the line, but accelerated very vigorously during cornering to reach $50 \mathrm{~km} / \mathrm{h}$ at the adaptive cruise control. However, this acceleration was too intense while driving in the curve, in terms of vehicle stability (close to the grip) and during the turn, the test car was too close to the vehicle ahead, requiring intervention by braking.

\section{Structure of research}

In the first step of the research the examination of the currently known automated vehicle functions have been carried out. Beside this some of the accidents related to automated driving assistant systems have been investigated. During the further investigations the Active Distance Cruise Control (ACC) system is going to be analyzed as one of the mostly used automated driving assistant system. At the same time there are many well-known constraints and deficiencies related to these systems. The practical experiences related to the mentioned defects provide a good basis for the further analysis targeting to identify those driving circumstances and characteristics where the efficiency of the driving assistant system is not sufficient.

In the next step the previously identified failure modes have been classified according to the methodology of the analysis. After that, the most important components of the required test process have been defined based on the structure of the related Euro NCAP test (Schram et al., 2013).
That has been followed by implementation of the test method. The measurement has been carried out on a 2018 middle-class passenger car. The model representation of the investigated ACC system has been based on the data collected during the test. Based on the measurement results, it is possible to identify separate operation ranges with different reliability.

The measurements has been performed based on the preliminary measurement plan. The test field of the measurement is located in Kistarcsa (Hungary), in a closed car park. The test took place on 18.10.2018 under night conditions, under artificial lighting and good visibility. According to the original plans, in some cases, the other vehicle would have been simulated by a large car-shaped cardboard box. The test car has been equipped with ACC Plus, so the system can stop and then start again, the cruise sensor is provided by the radar located in the middle of the front of the car.

\section{Approaching a stationary car from behind with operating ACC}

During the first measurement, the main objective was to simulate a stationary car with a car-sized paper box, but at $30 \mathrm{~km} / \mathrm{h}$ the ego car has not perceived the object, so the driver had to intervene the control process (Fildes et al., 2015).

In the next step the vehicle dummy was equipped with number plate to improve perceptibility. However, the previously introduced case has been repeated, since in neither case did the active distance cruise control system intervene the driving process. Finally, a real car was used during the measurement, but still no deceleration or braking was actuated by the driving assistant system, while the ACC has been set to the most sensitive mode (normal driving mode and early braking).

Accordingly it can be concluded that the system of the tested 2018 middle-class passenger car did not recognize either the vehicle shaped paper-box object or the real car. On the other hand the system operated with acceptable efficiency in case of moving vehicle detection (Biron and Pisu, 2015).

\section{Approaching a car moving at a constant speed from behind with operating ACC}

During the second measurement, the test vehicle's ACC was set at $30 \mathrm{~km} / \mathrm{h}$, while the vehicle in front of the ego car travelled at a speed of $20 \mathrm{~km} / \mathrm{h}$. This test has been carried out with several settings from early braking to late, and also with the sporty driving mode, but even in this case no 
malfunction was experienced, the system worked properly and reacted quickly. From the recorded data, it is clear that the system is able to maintain an adequate speed level in case of this test (Németh et al., 2013).

\section{Approaching a decelerating vehicle from behind with operating ACC}

In this test phase, it was investigated whether the car stops after continuous tracking if the vehicle in front of it stops. The speed was also set to $30 \mathrm{~km} / \mathrm{h}$ then the first car started braking operation (Figs. 1 and 2).

As it is presented by Figs. 1 and 2, the ego vehicle stopped at $0.27 \mathrm{~km}$ after the start of the measurement. The measurement was then carried out, with several ACC

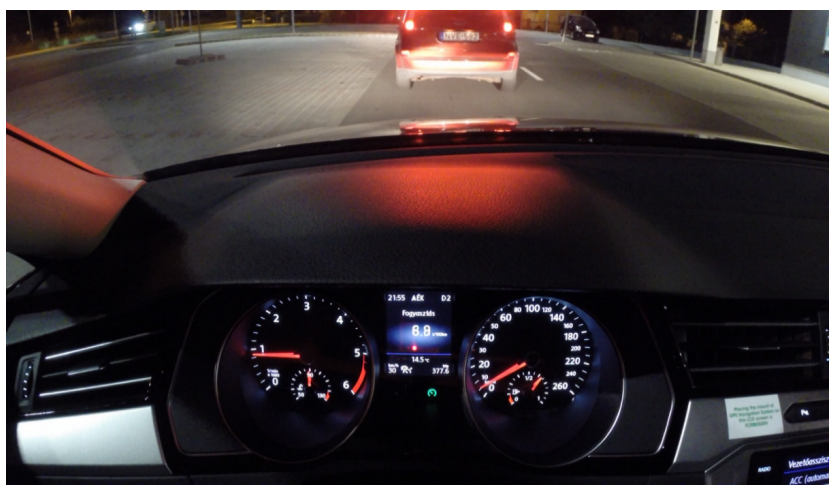

Fig. 1 Tracking a moving vehicle and stopping behind it settings, even with the most critical setting, the system could not be confused, always stopped properly.

\section{Different overlap situations, approaching a decelerating car with operating ACC}

The effectiveness of the system has been examined in 7 different cases:

\section{Braking until stop:}

Firstly, the case of approaching a parking vehicle was investigated, during the previous measurement the system performed well in case of $100 \%$ overlapping, then we reduced the degree of overlapping (Fig. 3).

2. $75 \%$ overlapping:

The measurement was performed $3+1$ times one after the other with a $75 \%$ overlapping. The first measurement failed because the ego vehicle had braked in the curve before the ACC was able to calibrate itself in the straight line section. Due to this failure the car could not be stopped, hence preventive intervention was needed to be made by the test driver. However, after this, all the next three measurements were performed flawlessly. Although the sample size was considerably small, the performed investigations have proved that the system performs well in case of the defined conditions.

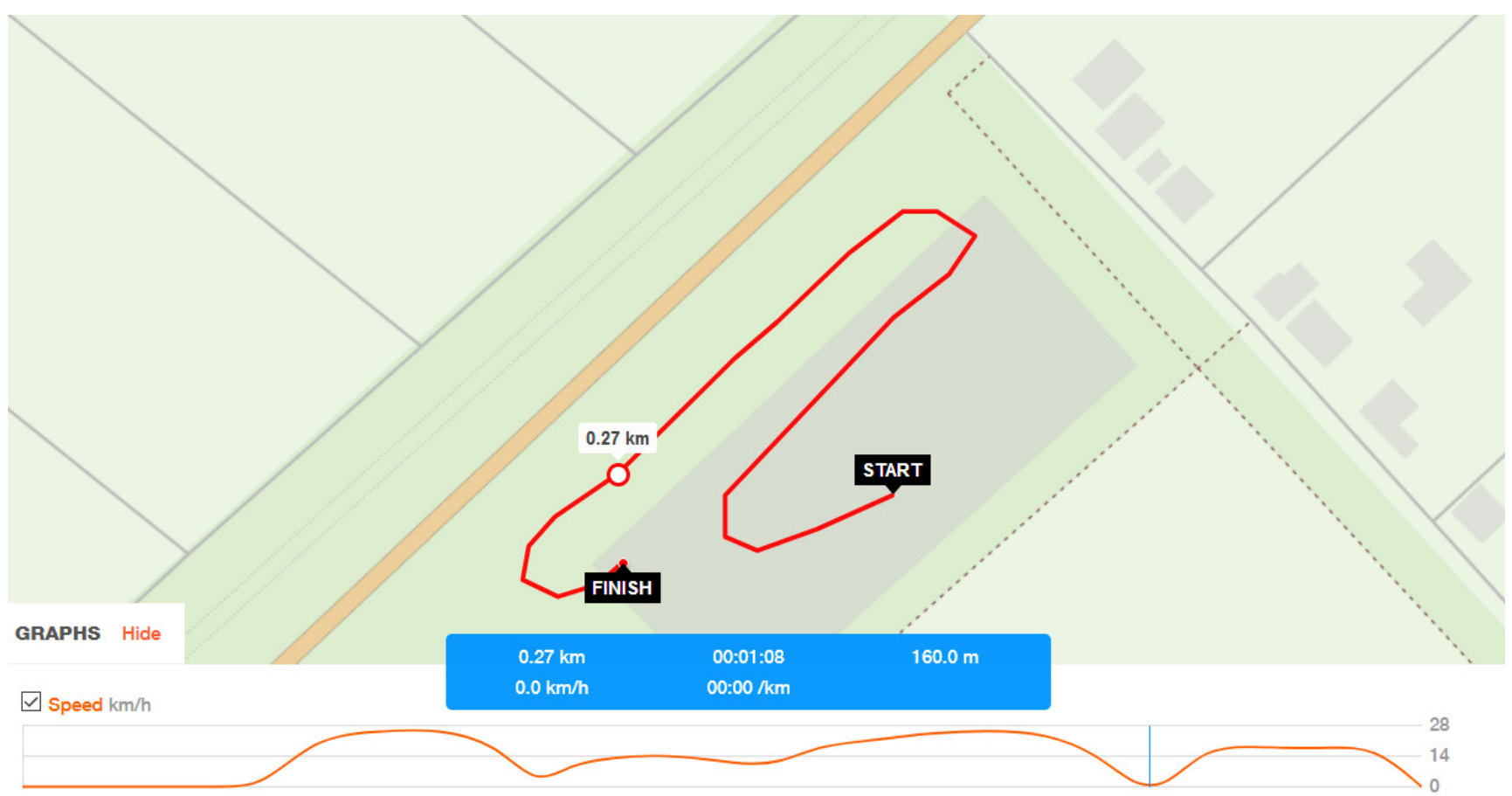

Fig. 2 Tracking a moving vehicle and stopping behind it 


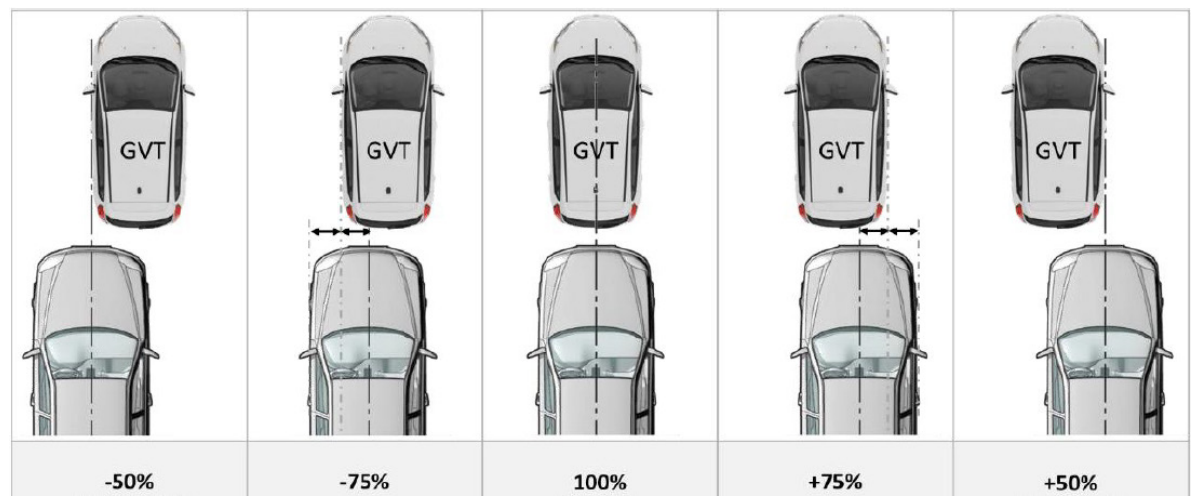

Fig. 3 Different overlapping (Test Site Sweden, online)

\section{3. $50 \%$ overlapping:}

In case of $50 \%$ overlapping, based on the performed 3 measurements, it can be concluded that the system worked perfectly and stopped in every necessary case, no intervention or maneuver was performed.

4. 25\% overlapping:

In the case of this measurement type, the sample size was extended to 5 measurements to achieve more reliable details related to operation efficiency of the system. From the 5 performed measurements, the system worked perfectly in 4 cases however the driver needed to perform intervention in 1 case. In this case the applied methodology differs from the Euro NCAP model, since they do not decrease the level of overlapping under $50 \%$, however according to our experiences, in real traffic conditions it is not uncommon to meet such situations.

\section{10\% overlapping:}

$10 \%$ overlapping was also simulated, where the edge of the left front wheel of the rear car was aligned with the inner line of the right rear wheel of the front car. In this case the sample included 5 elements as well. The situation was critical in 2 cases, but since the overlapping was marginal, a small steering was enough to avoid conflict. Accordingly, the average operation efficiency is estimated to $60 \%$. After that, most critical situation was investigated with the $10 \%$ overlapping, by continuously changing the level of overlapping (Ghadi and Török, 2019a).

6. $10 \%$ unsteady overlapping:

Since this scenario was a rather dangerous measurement, the rear car was the vehicle which was responsible for setting the level of overlapping. This approach could contribute to avoiding possible accidents. Finally, this scenario has resulted in the worst indicators, since out of 5 cases only 2 cases were successful. Accordingly, in the case of the identified conditions (10\%, unsteady overlapping) the operation efficiency of the system is estimated to be only $40 \%$. There were cases where the test vehicle was needed to be braked, since the frontal road surface was perceived empty and the test vehicle accelerated inordinately. On the other hand, there were cases where the system slowed down, however, when the test vehicle approached the front car, the sensor no longer detected the target and the test vehicle started to accelerate. Due to his, the driver needed to perform intervention (Rajamani, 2012) (Fig. 4).

\section{Pedestrian steps in front of a car which using ACC}

In the case of this measurement, a vehicle-pedestrian conflict was investigated. The pedestrian was modelled by a large rubber ball rolled in front of a car, crossing its path.

In this test 3 measurements were performed and in all three cases the vehicle drove through the ball, so it could be stated that it has not sensed it (Ghadi and Török, 2019b).

\section{Entering the roundabout after another car}

The roundabout was reached following a straight road section. Accordingly, the ACC was set to $90 \mathrm{~km} / \mathrm{h}$ (Fig. 5).

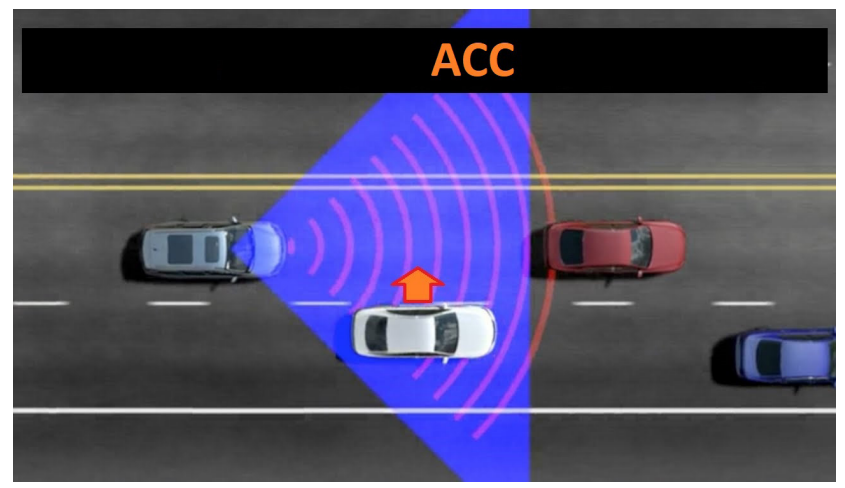

Fig. 4 Cutting in front of ACC (Engineering Explained, online) 


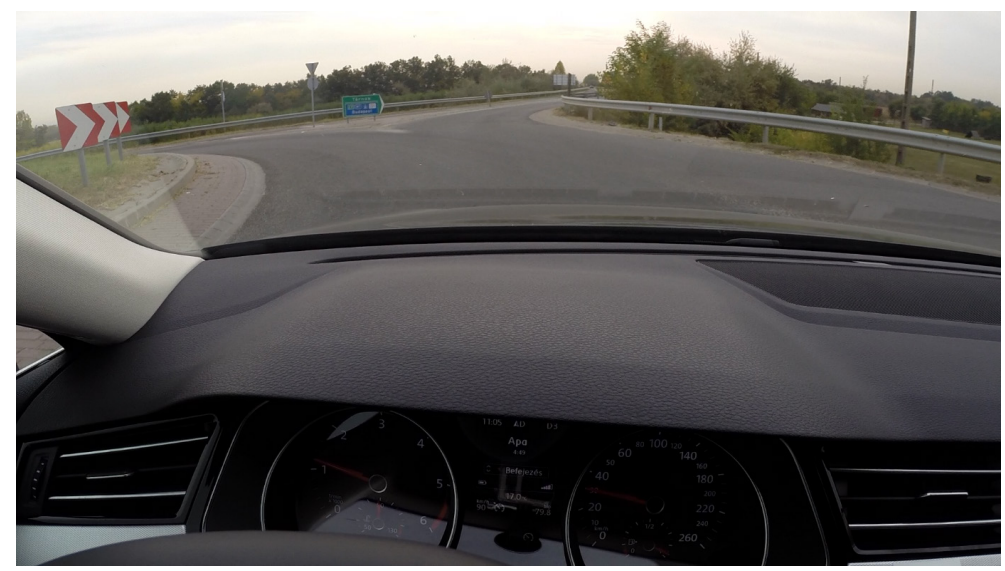

Fig. 5 In roundabout with ACC $(90 \mathrm{~km} / \mathrm{h})$

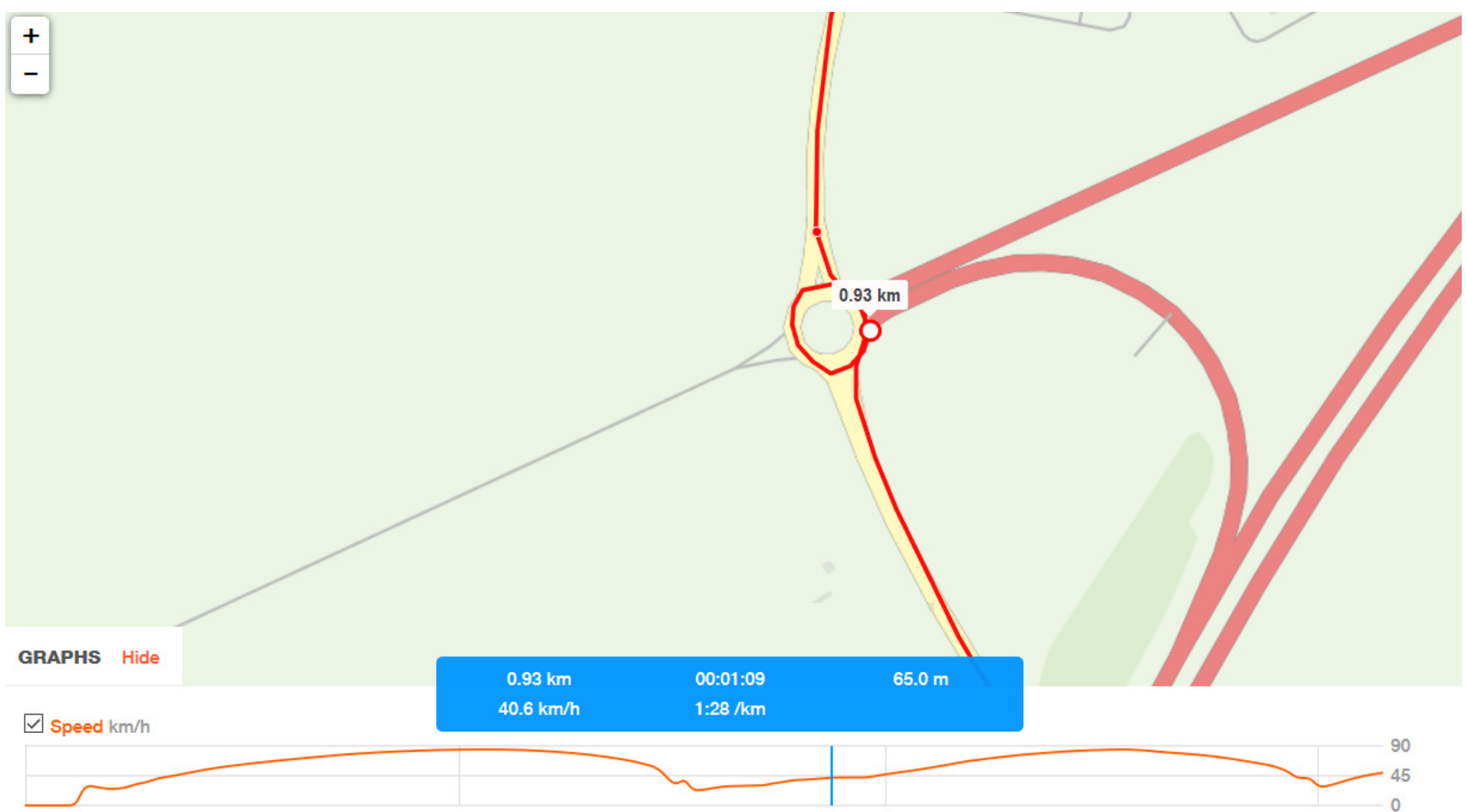

Fig. 6 In roundabout with ACC $(40 \mathrm{~km} / \mathrm{h})$

Before reaching the roundabout, the test vehicle stopped behind the front car. As soon as the front car had left the roundabout and the road surface had been empty, the test vehicle accelerated to $40 \mathrm{~km} / \mathrm{h}$ (Derenda et al., 2018; Török, 2017) (Fig. 6).

\section{Measurement experiences}

The table below presents a simplified analysis related to the operation efficiency of the investigated function (see in Table 1).

Since the lane change operation was a considerably dangerous process, the most critical cases were examined. It can be observed that the less the overlapping is the more
Table 1 ACC operation efficiency in different overlapping situations

\begin{tabular}{lccccc}
\hline \multicolumn{5}{c}{ Operation efficiency } \\
\hline $\begin{array}{l}\text { Overlapping } \\
\text { Lane changing with }\end{array}$ & 100 & 75 & 50 & 25 & 10 \\
ACC & $100 \%$ & - & - & - & $40 \%$ \\
$\begin{array}{l}\text { Vehicle following and } \\
\text { stop with ACC }\end{array}$ & $100 \%$ & $100 \%$ & $100 \%$ & $80 \%$ & $60 \%$ \\
Static object & $0 \%$ & - & - & - & - \\
\hline
\end{tabular}

significant reduction in the ACC operation efficiency can be provided (Wang et al., 2018).

It is important to emphasize that during the measurements, some of the boundaries of the ACC system identified by the manufacturer were step over and the vehicle was 
tested in situations that do not meet the predefined operation conditions identified by the producer. Accordingly, in the operating instructions, it is stated that the automated driving assistance function can only be operated in a safe way if the predefined conditions are given. However it also needs to be mentioned that the average driver may not have the ability and the competency to react properly if the traffic conditions immediately change. Accordingly, automated driving assistance functions may have the risk of reducing the awareness of the driver and bringing her/him in such unexpected situations where the abilities and competencies of an average driver may not be sufficient to control the occurred hazardous situation (Vujic et al., 2019).

In summary, the presented data illustrates the estimated performance of the ACC system in case of the lack of human intervention. So it can be seen that in these cases the efficiency of the system is not satisfactory. In addition to the above introduced considerations, it is advisable to summarize the development process of the ACC system model and the methodological framework for measuring system efficiency. In accordance with this, the model development process for evaluating system efficiency is illustrated in Fig. 7.

\section{SWOT analysis}

On the basis of the measurements and previous experience, a SWOT analysis of the ACC has been performed. In this analysis, the strengths, weaknesses, opportunities and threats of the system have been evaluated.

\section{Strengths:}

Tracking a preceding car with $100 \%$ overlapping along a straight track.

2. Weaknesses:

Tracking a preceding car with less than $50 \%$ overlapping or during a lane changing operation. Tracking in curve. Reaching parking vehicles/ other static objects.

\section{Opportunities:}

More information can be collected by installing a camera-based detection system in addition to the radar used in the test car. The coordinated operation of the fusion based sensing systems allows the environment perception module to be more accurate. This can significantly improve the operation efficiency, even in critical situations.

\section{Dangers:}

Critical situations, which require the intervention of the driver .If the driver gets used to relying on the ACC system, his/her attention may decrease. In critical situations, the inadequate recognition of emergency can lead to accident (Rudin-Brown and Parker, 2004).

\section{Summary}

In this article relevant test cases have been introduced and implemented derived from errors and failures experienced during previous operation of the system. During the research GNSS data and image files have been

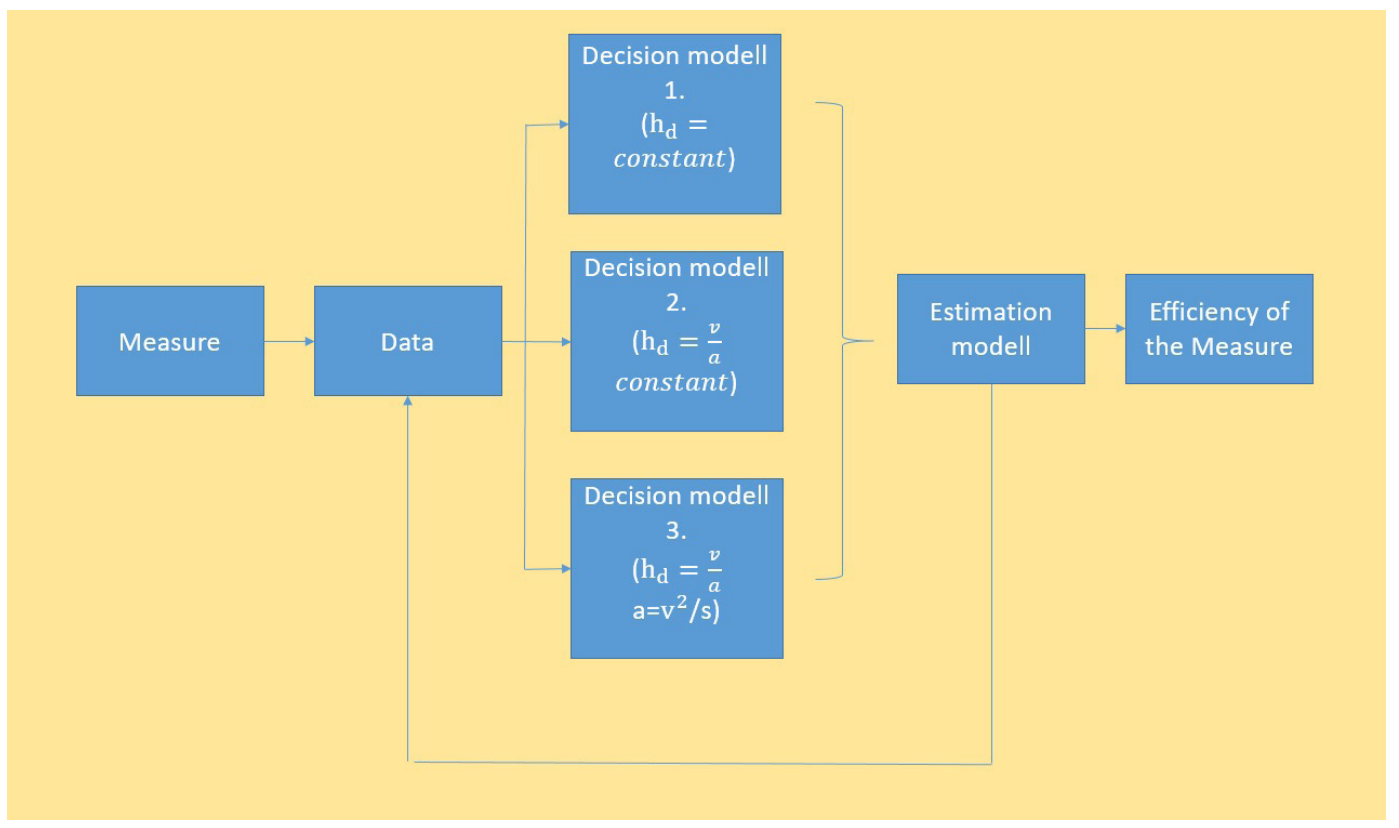

Fig. 7 Modell of ACC 
used to characterize and evaluate the analyzed cases. Accordingly the decision process has been considered as a black box, which has made it possible to focus on the relationship of input and output data. Therefore, the ACC system model proposed by the literature has been adapted to the conditions of the identified problem, especially considering the examined low-speed operation interval. Based on the achieved results it was possible to examine the performance of the ACC system. According to the reached outcomes, it can be concluded that only if the system has the required time to detect the target object, can the ACC module function properly. Furthermore, the system proved to be unreliable during some relevant traffic maneuvers (i.e. lane changing, driving in curve).

\section{References}

Biron, Z. A., Pisu, P. (2015) "Distributed Fault Detection and Estimation for Cooperative Adaptive Cruise Control System in a Platoon", In: Annual Conference of the Prognostics and Health Management Society 2015, Coronado, CA, USA, pp. 1-6. https://doi.org/10.36001/phmconf.2015.v7i1.2545

Derenda, T., Zanne, M., Zoldy, M., Torok, A. (2018) "Automatization in road transport: a review", Production Engineering Archives, 20(20), pp. 3-7.

Engineering Explained "How Adaptive Cruise Control Works - Step One For Autonomous Cars", [video online] Available at: youtube.com/ watch? $=$ IMYi3G7dkU4 [Accessed: 08 February 2020]

Fildes, B., Keall, M., Bos, N., Lie, A., Page, Y., Pastor, C., Pennisi, L., Rizzi, M., Thomas, P., Tingvall, C. (2015) "Effectiveness of low speed autonomous emergency braking in real-world rear-end crashes", Accident Analysis \& Prevention, 81, pp. 24-29. https://doi.org/10.1016/j.aap.2015.03.029

Galba, T., Solic, K., Lukic, I. (2015) "An Information Security and Privacy Self-Assessment (ISPSA) Tool for Internet Users", Acta Polytechnica Hungarica, 12(7), pp. 149-162.

Ghadi, M. Q., Török, Á. (2019a) "Comparison of Different Road Segmentation Methods", Promet - Traffic \& Transportation, 31(2), pp. $163-172$.

https://doi.org/10.7307/ptt.v31i2.2937

Ghadi, M., Török, Á. (2019b) "A comparative analysis of black spot identification methods and road accident segmentation methods", Accident Analysis \& Prevention, 128, pp. 1-7.

https://doi.org/10.1016/j.aap.2019.03.002

Kesting, A., Treiber, M., Schönhof, M., Helbing, D. (2007) "Extending Adaptive Cruise Control to Adaptive Driving Strategies", Transportation Research Record: Journal of the Transportation Research Board, 2000(1), pp. 16-24.

https://doi.org/10.3141/2000-03

Mandžuka, S. (2018) "Cooperative Systems in Traffic Technology and Transport", In: International Conference "New Technologies, Development and Applications", Sarajevo, Bosnia and Herzegovina, pp. 299-308.

https://doi.org/10.1007/978-3-319-90893-9_36
Therefore, the driver has to continuously pay attention for the driving process and has to be able to perform intervention whenever it is necessary. The importance of the study and the relevancy of the critical situations experienced during the investigations, are supported by the permanently developing Euro NCAP test methodological framework and the related results. Summing up it can be concluded that certain use cases supported by adaptive cruise control can be outstandingly critical, especially considering driving in curve or with small proportioned overlapping. So the development of these systems are still a major challenge for developers to obtain safe and fully autonomous vehicles (Kesting et al., 2007).

Németh, B., Csikós, A. A., Gáspár, P., Varga, I. (2013) "Multicriteria cruise control design considering geographic and traffic conditions", Acta Polytechnica Hungarica, 10(6), pp. 119-134.

Rajamani, R. (2012) "Vehicle Dynamics and Control", Springer, Boston, MA, USA. https://doi.org/10.1007/978-1-4614-1433-9

Rudin-Brown, C. M., Parker, H. A. (2004) "Behavioural adaptation to adaptive cruise control (ACC): implications for preventive strategies", Transportation Research Part F: Traffic Psychology and Behaviour, 7(2), pp. 59-76. https://doi.org/10.1016/j.trf.2004.02.001

Schram, R., Williams, A., van Ratingen, M. (2013) "Implementation of Autonomous Emergency Braking (AEB), the Next Step in Euro NCAP'S Safety Assessment", ESV, Seoul, South Korea, Paper number: 13-0269.

Test Site Sweden "Euro NCAP Asta Zerotesters 2017", [pdf] Test Site Sweden, Available at: https:/www.testsitesweden.com/sites/ default/files/content/euro_ncap_asta_zerotesters_day_2017-0425.pdf [Accessed: 19 December 2018]

Török, Á. (2017) "Comparative analysis between the theories of road transport safety and emission", Transport, 32(2), pp. 192-197. https://doi.org/10.3846/16484142.2015.1062798

Vujic, M., Mandzuka, S., Dedic, L. (2019) "IoT Concept in Cooperative Traffic Management", In: International Conference "New Technologies, Development and Applications", Sarajevo, Bosnia and Herzegovina, pp. 406-410. https://doi.org/10.1007/978-3-030-18072-0_47

Wang, M., Hoogendoorn, S. P., Daamen, W., van Arem, B., Shyrokau, B., Happee, R. (2018) "Delay-compensating strategy to enhance string stability of adaptive cruise controlled vehicles", Transportmetrica B: Transport Dynamics, 6(3), pp. 211-229. https://doi.org/10.1080/21680566.2016.1266973 